\title{
Out-of-equilibrium dynamics of topological and anomalous effects from chiral kinetic theory
}

\author{
Niklas Mueller* \\ Physics Department, Brookhaven National Laboratory, Bldg. 510A, Upton, NY 11973, USA \\ E-mail: nmuellerabnl.gov
}

\begin{abstract}
Recent studies suggest that important contributions to the Chiral Magnetic Effect (CME) originate in the pre-equilibrium phase of a collision. While real-time lattice simulations can be utilized to understand the dynamics of anomalous effects in the earliest stages of a collision, quantitative predictions of experimental signatures are only feasible once the subsequent transport of the messengers of the CME through the fireball are understood. This motivates the need of a Chiral Kinetic Theory for relativistic fermions. In this talk we present a novel approach based on the world line formulation of quantum field theory that clarifies the relative role of a possible Berry phase and chiral anomaly that generates topological transitions. Our formulation is Lorentz covariant and independent of adiabatic approximations. Our framework allows us to follow ab initio the fate of the Chiral Magnetic current from the earliest times (via solutions of the Dirac equation in topological sphaleron backgrounds) through its matching to Chiral Kinetic Theory and finally to Chiral Magneto-Hydrodynamics. We discuss the implications of our results for quantitative extraction of the CME in heavy-ion collisions.
\end{abstract}

XIII Quark Confinement and the Hadron Spectrum - Confinement2018

31 July - 6 August 2018

Maynooth University, Ireland

${ }^{*}$ Speaker. 


\section{Introduction}

Searches for observable consequences of topology-changing sphaleron transition in QuantumChromodynamics (QCD) [1,2] have attracted great interest. In the context of ultra-relativistic heavy-ion collisions, a striking manifestation is the proposed Chiral Magnetic Effect (CME) [3, 4] where electric currents are generated along strong magnetic fields by means of the chiral anomaly. Experimental and theoretical interest in chiral transport phenomena is not limited to high energy and nuclear physics, but ranges from condensed matter [5, 6] to astrophysics [7, 8] and beyond.

Recent heavy ion experiments are not yet conclusive concerning the existence of the CME [9, $10,11]$. This is in part due short life-time of the strong magnetic fields created in ion-ion collisions, indicating that the origin of these anomalous phenomena is in the pre-equilibrium phase of the collision. Here, theoretical descriptions of the non-equilibrium quantum-many-body dynamics, which consistently include the effects of topological transitions and of the chiral anomaly, are challenging. Ab-initio descriptions of anomalous and topological effects are available at the earliest moments of a collision, where matter is in a strongly correlated "Glasma"-state, dominated by large gluon occupancies. In this regime, the real-time dynamics can be described by classical-statistical lattice simulations $[12,13,14]$, which can be systematically computed within the Color Glass Condensate effective theory $[15,16,17]$. Here, one relies on a non-perturbatively large phase space occupancy of gluons in the weak-coupling limit $\left(\alpha_{s} \ll 1\right)$ to approximate the early time dynamics by a statistical ensemble of classical Yang-Mills fields [18, 19, 20].

In this limit, the real time dynamics of fermions, too, can be studied from first principles by numerically solving the operator Dirac equation for a given gauge field background. In $[12,13$, 14] we presented a first step towards quantitative descriptions of early-time anomalous transport phenomena, by studying the generation of vector and chiral charges during a topological sphaleron transition in a background magnetic field.

Describing the space-time evolution of the CME at later times is more challenging: As the fireball expands, gluon occupation numbers drop to unity and classical-statistical simulation techniques break down. Here, simulation results must be matched to effective quantum kinetic theory $[21,22]$. The development of a Boltzmann transport equation within such transport framework is an important ingredient for systematic analysis of CME phenomenology.

The formulation of a chiral kinetic theory requires to consistently incorporate internal degrees of freedom, such as color and spin, in a phase space formulation. Specifically, conservation and anomalous non-conservation of fermionic current by means of the chiral anomaly, as well as interactions of chiral probes with topological and non-topological gauge field fluctuations must be understood. Many suggestions for such a chiral kinetic theory have been put forward, e.g. including a point particle action with a Berry monopole term [23, 24], Wigner functions [27] or effective theories [25, 26] or hydrodynamic descriptions [28, 29, 30].

In this talk we present an overview over recent progress $[31,32,33]$ in deriving a consistent quantum kinetic theory for chiral fermions from the world-line approach to quantum field theory $[34,35,36,37]$. Here, an important ingredient is the realization of internal symmetries via anti-commuting coordinates, with the representation of spin via Grassmann variables going back to seminal work by Berezin and Marinov [38]. The outline of this manuscript is as follows: In section 2 we will give a short introduction into the world-line representation of one-loop effective 
actions for fermions. In euclidean formulation we demonstrate the emergence of the chiral anomaly from the imaginary part of the effective action, related to the emergence of fermionic zero modes. In section 3 we then demonstrate the emergence of a Berry phase in the non-relativistic limit of our framework. We show that the Berry phase is unrelated to the chiral anomaly. In section 4, we outline elements of a generalized phase space construction including spin as anti-commuting coordinates.

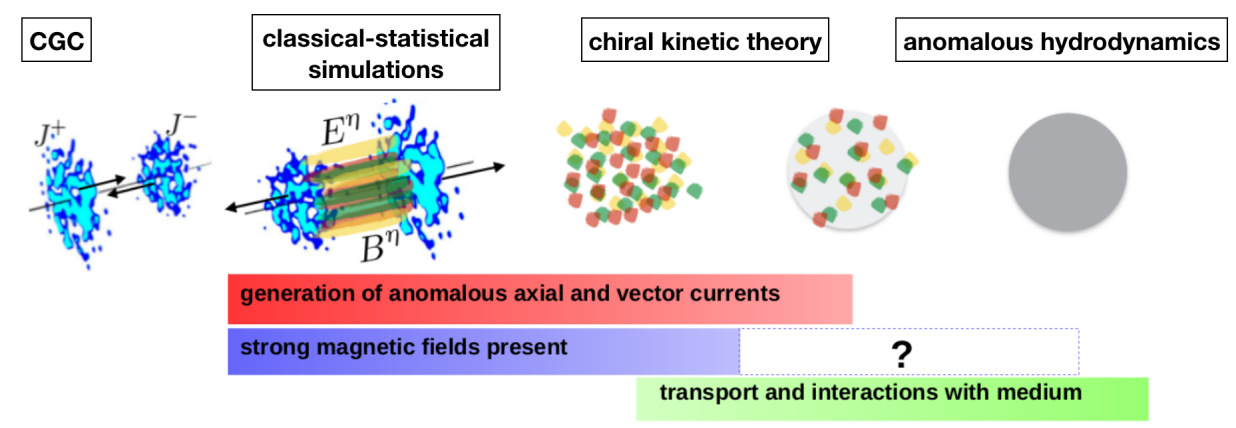

Figure 1: Schematic overview over the space-time evolution of an ultra-relativistic heavy-ion collision and the relevant stages for anomalous transport. At early times, strong magnetic fields are present and anomalous axial and vector currents are created. Based on the Color Glass Condensate framework [15, 16], the dynamics of gluons and fermions can be studied with classical statistical simulations [31, 32, 33]. Subsequently, the fireball created in the collision becomes dilute and classical-statistical approximations break down. Here, simulation results might serve as initial conditions for an effective quantum kinetic theory, including internal symmetries such as spin and color $[31,32,33]$. At late times, the system can be described using anomalous hydrodynamics. Figure taken from [14].

\section{World-line representation of one-loop effective actions}

In this section we give a short overview over the world-line representation for one-loop effective actions in quantum field theory [34, 35, 36, 37]. For simplicity, we consider the QED action for fermions coupled to background vector and axial-vector gauge fields ${ }^{1}, S[A, B]=\int d^{4} x \bar{\psi}[i \not \partial+$ $\left.A+\gamma_{5} \not B\right] \psi$. The fermionic part of the path integral yields the fermion one loop effective action,

$$
W[A, B] \equiv \log \operatorname{det}(i \not \partial+\not A+\not B)=W_{R}[A, B]+i W_{I}[A, B] .
$$

Here, both the real and imaginary part of the effective action have a heat-kernel expression, which reads for the real part

$$
W_{\mathbb{R}}=\frac{1}{8} \int_{0}^{\infty} \frac{d T}{T} \mathscr{N} \int_{P} \mathscr{D} x \int_{A P} \mathscr{D} \psi \operatorname{tr} \exp \left\{-\int_{0}^{T} d \tau \mathscr{L}(\tau)\right\},
$$

\footnotetext{
${ }^{1}$ The latter is understood as a auxiliary variational parameter.
} 
which includes the world-line Lagrangian, $\mathscr{L}=\operatorname{diag}\left(\mathscr{L}_{L}, \mathscr{L}_{R}\right)$, where $L / R$ denote left and right chiralities, given by

$$
\mathscr{L}_{L / R}=\frac{\dot{x}^{2}}{2 \varepsilon}+\frac{1}{2} \psi_{a} \dot{\psi}_{a}-i \dot{x}_{\mu}(A \pm B)_{\mu}-\frac{i \varepsilon}{2} \psi_{\mu} \psi_{v} F_{\mu \nu}[A \pm B],
$$

where $\psi_{a}$ are the Wigner-Weyl-symbols of $8 \times 8$ Dirac $\gamma$ matrices with index $a=1, \cdots, 6$ and $\varepsilon$ is a positive constant ('einbein') encoding reparametrization-invariance of the world-line parameter $\tau$. Eq.(2.2) can be interpreted as a quantum mechanical path integral of point particles $x^{\mu}$ and associated Grassmann degrees of freedom for spin $\psi^{a}$, quantized on a circle with periodic and anti-periodic boundary conditions, respectively.

As discussed in [39], a manifestation of the chiral anomaly is that the imaginary part of the fermionic effective action is ill-defined. In the world-line framework a heat kernel expression for the imaginary part can only be found by explicitly violating chiral symmetry. In practice the world-line expression for the imaginary part closely resembles that for the real-part, containing the similar single-particle action Eq.(2.3), albeit with the axial-vector gauge field multiplied with a parameter $B \rightarrow \alpha B$, which is integrated over. Moreover, the imaginary part contains a world-line insertion, resulting in periodic boundary conditions for the anti-commuting spin variables and in the emergence of fermionic zero modes. The anomaly equation can then be directly derived from the imaginary part by variation wrt. the auxiliary $B_{\mu}$,

$$
\left.\partial_{\mu}\left\langle j_{\mu}^{5}(y)\right\rangle \equiv \partial_{\mu} \frac{i \delta W_{\mathbb{I}}}{\delta B_{\mu}(y)}\right|_{B=0}=-\frac{1}{16 \pi^{2}} \varepsilon^{\mu v \rho \sigma} F_{\mu v}(y) F_{\rho \sigma}(y) .
$$

and setting $B_{\mu}=0$. Details of this derivation can be found in [31, 32].

\section{The emergence of Berry's phase}

In this section we show that the real-part of the world-line effective action, presented in section 2, contains a Berry phase in the non-relativistic and adiabatic limit. Towards, this end, we continue the world-line Lagrangian, Eq.(2.3) to Minkowskian metric $g=\operatorname{diag}(-,+,+,+)$ and we further include a constraint $\frac{\dot{x}_{\mu} \psi^{\mu}}{2 \mathscr{E}}+m \psi_{5}=0$ to remove unphysical degrees from the spectrum ${ }^{2}$. For massive fermions and in proper time gauge $\tau=c t \sqrt{1-(\mathbf{v} / c)^{2}}, \sqrt{-\dot{z}^{2}}=1$, where $\dot{z}^{2}=\left(d x^{\mu} / d \tau\right)^{2}$, the nonrelativistic action is $S=\int d \tau \mathscr{L}=\int d t c \sqrt{1-(\mathbf{v} / c)^{2}} \mathscr{L}$, with $\mathbf{v}=d \mathbf{x} / d t$. At $O\left(\mathbf{v} / c^{2}\right)$ this can be written as

$\mathscr{L}_{N R}=-m c^{2}+\frac{1}{2} m \mathbf{v}^{2}+\frac{i}{2}\left(\psi_{i} \dot{\psi}_{i}-\psi_{0} \dot{\psi}_{0}\right)-A^{0}+\frac{\mathbf{v}}{c} \cdot \mathbf{A}+\frac{\mathbf{S} \cdot\left(\left[\mathbf{v} / c-\mathbf{A} /\left(m c^{2}\right)\right] \times \mathbf{E}\right)}{m c}+\frac{\mathbf{S} \cdot \mathbf{B}}{m}+O\left(\frac{\mathbf{v}^{3}}{c^{3}}\right)$,

where we have now changed notation in accordance with the Minkowskian metric $\psi_{4} \rightarrow \psi_{0}$ and where $i=1,2,3$, with a corresponding Hamiltonian

$$
H \equiv m c^{2}+\frac{\left(\mathbf{p}-\frac{\mathbf{A}}{c}\right)^{2}}{2 m}+A^{0}(x)-\frac{\mathbf{S} \cdot\left(\left[\mathbf{v} / c-\mathbf{A} /\left(m c^{2}\right)\right] \times \mathbf{E}\right)}{2 m c}-\frac{\mathbf{B} \cdot \mathbf{S}}{m} .
$$

\footnotetext{
${ }^{2}$ In particular $\psi_{6}$ is not dynamical and can be integrated out, while $\psi_{5}$ can be eliminated by imposing this constraint.
} 
In the adiabatic limit, transitions between spin states are suppressed and correspondingly the real part of the world line effective action can be written as

$$
W_{\mathbb{R}}=\int \mathscr{D} x \mathscr{D} p \exp \left(i \int d t[\dot{\mathbf{x}} \cdot \mathbf{p}-\tilde{H}]\right),
$$

with $\tilde{H}=m c^{2}+\frac{(\mathbf{p}-A / c)^{2}}{2 m}+A^{0}(x)-\dot{\mathbf{p}} \cdot \mathscr{A}(\mathbf{p})$. The path integral in this limit contains a Berry phase $\mathscr{A}(\mathbf{p}) \equiv-i\left\langle\psi^{+}(\mathbf{p})\left|\nabla_{p}\right| \psi^{+}(\mathbf{p})\right\rangle$. We contrast our results to that of [23, 24], where the topology of the chiral anomaly was identified with a Berry phase. Our derivation suggests a different interpretation: Here, the anomaly is tied to the emergence of fermionic zero modes and to the imaginary part of the effective action, while a Berry phase is found for the real part - indicating that anomaly and Berry phase are not related. Our findings thus agree with observations made by Fujikawa in other context [40, 41].

\section{Elements of Chiral Kinetic Theory}

Starting from the saddle-point limit of the Schwinger-Keldysh world-line path integral, one obtains a classical phase space formulation, where spin is expressed by anti-commuting Grassmann coordinates. Here the central object is a Liouville equation,

$$
0=\{f, H\}=f\left(\frac{\overleftarrow{\partial}}{\partial x^{\mu}} \dot{x}^{\mu}+\frac{\overleftarrow{\partial}}{\partial P^{\mu}} \dot{P}^{\mu}+\frac{\overleftarrow{\partial}}{\partial \psi^{\mu}} \dot{\psi}^{\mu}+\frac{\overleftarrow{\partial}}{\partial \psi_{5}} \dot{\psi}_{5}\right)
$$

where the fundamental Poisson brackets are given by $\left\{x^{\mu}, p_{v}\right\}=\delta_{v}^{\mu},\left\{\psi^{\mu}, \psi_{v}\right\}=-i \delta_{v}^{\mu},\left\{\psi_{5}, \psi_{5}\right\}=$ $-i$ and $\left\{\psi^{\mu}, \psi_{5}\right\}=0$. The world-line Hamiltonian in the massive case is given by

$$
H=\frac{\varepsilon}{2}\left(P^{2}+m^{2}+i \psi^{\mu} F_{\mu \nu} \psi^{v}\right)+\frac{i}{2}\left(P_{\mu} \psi^{\mu}+m \psi_{5}\right) \chi,
$$

and in the chiral case by

$$
H=\frac{\varepsilon}{2}\left(P^{2}+i \psi^{\mu} F_{\mu \nu} \psi^{v}\right)+\frac{i}{2}\left(P_{\mu} \psi^{\mu} \pm \frac{i}{3} \varepsilon^{\mu \nu \alpha \beta} P_{\mu} \psi_{\nu} \psi_{\alpha} \psi_{\beta}\right) \chi
$$

Here, $P^{\mu} \equiv p_{\mu}-A_{\mu}$. For a specific gauge choice, $\chi=0$ and $\varepsilon=1 / P^{0}$, the following equations of motion follow

$$
\begin{aligned}
\dot{P}^{\mu} & =v_{\alpha} F^{\mu \alpha}-\frac{i}{2 P^{0}} \psi^{\alpha} \partial^{\mu} F_{\alpha \beta} \psi^{\beta}, \\
\dot{x}^{\mu} & =v^{\mu} \\
\dot{\psi}^{\mu} & =\frac{1}{P^{0}} F^{\mu \alpha} \psi_{\alpha},
\end{aligned}
$$

where $v^{\mu}=\varepsilon P^{\mu}$ and $\dot{\psi}_{5}=0$ is not dynamical. The classical equations of motion for both massive and chiral case coincide. However, the equations are supplemented by spectral constraints which distinguish the two cases,

$$
\begin{aligned}
& P^{2}+m^{2}=0, \quad P_{\mu} \psi^{\mu}+m \psi_{5}=0 \quad \text { (massive case), } \\
& P^{2}=0, \quad P_{\mu} \psi^{\mu} \pm \frac{i}{3} \varepsilon^{\mu v \alpha \beta} P_{\mu} \psi_{v} \psi_{\alpha} \psi_{\beta}=0 \quad \text { (chiral case) }
\end{aligned}
$$


In order to derive a Boltzmann transport equation, we follow a stochastic approach whereby the microscopic Liouville density $f$ is written in terms of the one-particle Boltzmann distribution function, $f \equiv\langle f\rangle+\delta f=\bar{f}+\delta f$. The expectation value denotes an average wrt. a statistical ensemble specified by at some initial time through the initial density matrix in the SchwingerKeldysh path integral. Likewise, we can split the gauge sector into average and fluctuating fields, $A^{\mu} \equiv\left\langle A^{\mu}\right\rangle+\delta A^{\mu}=\bar{A}^{\mu}+\delta A^{\mu}$ and in turn split the Liouville equation. We obtain a Boltzmann equation,

$$
\bar{f}\left(\frac{\overleftarrow{\partial}}{\partial x^{\mu}}\left[\varepsilon P^{\mu}\right]+\frac{\overleftarrow{\partial}}{\partial P^{\mu}}\left[\varepsilon \bar{F}^{\mu \alpha} P_{\alpha}-\frac{i \varepsilon}{2} \psi^{\alpha} \partial^{\mu} \bar{F}_{\alpha \beta} \psi^{\beta}\right]+\frac{\overleftarrow{\partial}}{\partial \psi^{\mu}}\left[\varepsilon \bar{F}^{\mu \alpha} \psi_{\alpha}\right]\right)=C[\delta f, \delta F]
$$

where the collision term is given by

$$
C[\delta f, \delta F] \equiv-\varepsilon\left\langle\delta f \frac{\overleftarrow{\partial}}{\partial P^{\mu}} \delta F^{\mu \alpha}\right\rangle P_{\alpha}-\varepsilon\left\langle\delta f \frac{\overleftarrow{\partial}}{\partial \psi^{\mu}} \delta F^{\mu \alpha}\right\rangle \psi_{\alpha}+\frac{i \varepsilon}{2}\left\langle\delta f \frac{\overleftarrow{\partial}}{\partial P^{\mu}} \partial^{\mu} \delta F_{\alpha \beta}\right\rangle \psi^{\alpha} \psi^{\beta}
$$

Similarly, the equation for the fluctuations reads

$$
\delta f\left(\frac{\overleftarrow{\partial}}{\partial x^{\mu}}\left[\varepsilon P^{\mu}\right]+\frac{\overleftarrow{\partial}}{\partial P^{\mu}}\left[\varepsilon \bar{F}^{\mu \alpha} P_{\alpha}-\frac{i \varepsilon}{2} \psi^{\alpha} \partial^{\mu} \bar{F}_{\alpha \beta} \psi^{\beta}\right]+\frac{\overleftarrow{\partial}}{\partial \psi^{\mu}}\left[\varepsilon \bar{F}^{\mu \alpha} \psi_{\alpha}\right]\right)=K[\delta F]
$$

where

$$
K[\delta F] \equiv-\bar{f}\left(\frac{\overleftarrow{\partial}}{\partial P^{\mu}}\left[\varepsilon \delta F^{\mu \alpha} P_{\alpha}-\frac{i \varepsilon}{2} \psi^{\alpha} \partial^{\mu} \delta F_{\alpha \beta} \psi^{\beta}\right]+\frac{\overleftarrow{\partial}}{\partial \psi^{\mu}}\left[\varepsilon \delta F^{\mu \alpha} \psi_{\alpha}\right]\right)
$$

These equations form the starting point for a derivation of chiral kinematic theory. In practice Eq.(4.9) and Eq.(4.11) form an infinite hierarchy of equations for the one-particle distribution function as well as higher moments of the fluctuations $\langle\delta f \delta f\rangle,\langle\delta f \delta f \delta f\rangle, \ldots$ One must truncate these equations using a power counting scheme, in order to integrate out the fluctuations in closed form. Furthermore, the effects of the anomaly are contained in the SK path integral. Here, one must compute current expectation values inside the path integral before taking the saddle point limit [31, 32].

\section{Summary}

We presented a world-line approach to constructing an effective kinetic theory for relativistic fermions, via a heat-kernel regularization in Quantum Field Theory. In this approach the emergence of the chiral anomaly is related to the imaginary part of the fermionic effective action. A many-body generalization of the approach, via a Schwinger-Keldysh non-equilibrium path integral, allows to derive a semi-classical Liouville equation for phase space trajectories, generalized to include spin via Grassmann coordinates. Future application of the worldline approach may include the dynamics of topological transitions in non-Abelian plasmas, thereby treating the color group $S U(N)$ too using Grassman variables, or computing the structure of nuclei at high energies. 


\section{References}

[1] F. R. Klinkhamer and N. S. Manton, Phys. Rev. D 30, 2212 (1984).

[2] R. F. Dashen, B. Hasslacher and A. Neveu, Phys. Rev. D 10, 4138 (1974).

[3] D. E. Kharzeev, L. D. McLerran and H. J. Warringa, Nucl. Phys. A 803, 227 (2008) doi:10.1016/j.nuclphysa.2008.02.298 [arXiv:0711.0950 [hep-ph]].

[4] K. Fukushima, D. E. Kharzeev and H. J. Warringa, Phys. Rev. D 78, 074033 (2008) doi:10.1103/PhysRevD.78.074033 [arXiv:0808.3382 [hep-ph]].

[5] P. Hosur and X. Qi, Comptes Rendus Physique 14, 857 (2013) doi:10.1016/j.crhy.2013.10.010 [arXiv:1309.4464 [cond-mat.str-el]].

[6] E. V. Gorbar, V. A. Miransky, I. A. Shovkovy and P. O. Sukhachov, Low Temp. Phys. 44, no. 6, 487 (2018) [Fiz. Nizk. Temp. 44, 635] doi:10.1063/1.5037551 [arXiv:1712.08947 [cond-mat.mes-hall]].

[7] A. Boyarsky, J. Frohlich and O. Ruchayskiy, Phys. Rev. Lett. 108, 031301 (2012) doi:10.1103/PhysRevLett.108.031301 [arXiv:1109.3350 [astro-ph.CO]].

[8] S. Sen and N. Yamamoto, Phys. Rev. Lett. 118, no. 18, 181601 (2017) doi:10.1103/PhysRevLett.118.181601 [arXiv:1609.07030 [hep-th]].

[9] B. I. Abelev et al. [STAR Collaboration], Phys. Rev. Lett. 103, 251601 (2009) doi:10.1103/PhysRevLett.103.251601 [arXiv:0909.1739 [nucl-ex]].

[10] B. I. Abelev et al. [STAR Collaboration], Phys. Rev. C 81, 054908 (2010) doi:10.1103/PhysRevC.81.054908 [arXiv:0909.1717 [nucl-ex]].

[11] B. Müller and A. Schäfer, Phys. Rev. D 98, no. 7, 071902 (2018) doi:10.1103/PhysRevD.98.071902 [arXiv:1806.10907 [hep-ph]].

[12] N. Müller, S. Schlichting and S. Sharma, Phys. Rev. Lett. 117, no. 14, 142301 (2016) doi:10.1103/PhysRevLett.117.142301 [arXiv:1606.00342 [hep-ph]].

[13] M. Mace, N. Mueller, S. Schlichting and S. Sharma, Phys. Rev. D 95, no. 3, 036023 (2017) doi:10.1103/PhysRevD.95.036023 [arXiv:1612.02477 [hep-lat]].

[14] M. Mace, N. Mueller, S. Schlichting and S. Sharma, Nucl. Phys. A 967, 752 (2017) doi:10.1016/j.nuclphysa.2017.05.040 [arXiv:1704.05887 [hep-lat]].

[15] L. D. McLerran and R. Venugopalan, Phys. Rev. D 49, 2233 (1994) doi:10.1103/PhysRevD.49.2233 [hep-ph/9309289].

[16] L. D. McLerran and R. Venugopalan, Phys. Rev. D 49, 3352 (1994) doi:10.1103/PhysRevD.49.3352 [hep-ph/9311205].

[17] F. Gelis, E. Iancu, J. Jalilian-Marian and R. Venugopalan, Ann. Rev. Nucl. Part. Sci. 60, 463 (2010) doi:10.1146/annurev.nucl.010909.083629 [arXiv:1002.0333 [hep-ph]].

[18] A. Krasnitz and R. Venugopalan, Phys. Rev. Lett. 84, 4309 (2000) doi:10.1103/PhysRevLett.84.4309 [hep-ph/9909203].

[19] P. Romatschke and R. Venugopalan, Phys. Rev. Lett. 96, 062302 (2006) doi:10.1103/PhysRevLett.96.062302 [hep-ph/0510121].

[20] J. Berges, K. Boguslavski, S. Schlichting and R. Venugopalan, Phys. Rev. D 89, no. 7, 074011 (2014) doi:10.1103/PhysRevD.89.074011 [arXiv:1303.5650 [hep-ph]]. 
[21] R. Baier, A. H. Mueller, D. Schiff and D. T. Son, Phys. Lett. B 502, 51 (2001) doi:10.1016/S0370-2693(01)00191-5 [hep-ph/0009237].

[22] P. B. Arnold, G. D. Moore and L. G. Yaffe, JHEP 0011, 001 (2000) doi:10.1088/1126-6708/2000/11/001 [hep-ph/0010177].

[23] D. T. Son and N. Yamamoto,Phys. Rev. Lett. 109, 181602 (2012)doi:10.1103/PhysRevLett.109.181602[arXiv:1203.2697 [cond-mat.mes-hall]].

[24] M. A. Stephanov and Y. Yin, Phys. Rev. Lett. 109, 162001 (2012) doi:10.1103/PhysRevLett.109.162001 [arXiv:1207.0747 [hep-th]].

[25] C. Manuel and J. M. Torres-Rincon, Phys. Rev. D 90, no. 7, 076007 (2014) doi:10.1103/PhysRevD.90.076007 [arXiv:1404.6409 [hep-ph]].

[26] C. Manuel and J. M. Torres-Rincon, Phys. Rev. D 92, no. 7, 074018 (2015) doi:10.1103/PhysRevD.92.074018 [arXiv:1501.07608 [hep-ph]].

[27] J. H. Gao, Z. T. Liang, S. Pu, Q. Wang and X. N. Wang, Phys. Rev. Lett. 109, 232301 (2012) doi:10.1103/PhysRevLett.109.232301 [arXiv:1203.0725 [hep-ph]].

[28] D. T. Son and P. Surowka, Phys. Rev. Lett. 103, 191601 (2009) doi:10.1103/PhysRevLett.103.191601 [arXiv:0906.5044 [hep-th]].

[29] A. V. Sadofyev and M. V. Isachenkov, Phys. Lett. B 697, 404 (2011) doi:10.1016/j.physletb.2011.02.041 [arXiv:1010.1550 [hep-th]].

[30] P. Glorioso, H. Liu and S. Rajagopal, arXiv:1710.03768 [hep-th].

[31] N. Mueller and R. Venugopalan, Phys. Rev. D 97, no. 5, 051901 (2018) doi:10.1103/PhysRevD.97.051901 [arXiv:1701.03331 [hep-ph]].

[32] N. Mueller and R. Venugopalan, Phys. Rev. D 96, no. 1, 016023 (2017) doi:10.1103/PhysRevD.96.016023 [arXiv:1702.01233 [hep-ph]].

[33] N. Mueller and R. Venugopalan, PoS CPOD 2017, 047 (2018) doi:10.22323/1.311.0047 [arXiv:1712.04057 [hep-ph]].

[34] M. J. Strassler, Nucl. Phys. B 385, 145 (1992) [hep-ph/9205205].

[35] E. D’Hoker and D. G. Gagne, Nucl. Phys. B 467, 272 (1996) doi:10.1016/0550-3213(96)00125-3 [hep-th/9508131].

[36] E. D’Hoker and D. G. Gagne, Nucl. Phys. B 467, 297 (1996) doi:10.1016/0550-3213(96)00126-5 [hep-th/9512080].

[37] M. Mondragon, L. Nellen, M. G. Schmidt and C. Schubert, Phys. Lett. B 351, 200 (1995) [hep-th/9502125].

[38] F. A. Berezin and M. S. Marinov, ††Annals Phys. 104, 336 (1977). ††doi:10.1016/0003-4916(77)90335-9 ††

[39] L. Alvarez-Gaume and E. Witten, Nucl. Phys. B 234, 269 (1984). doi:10.1016/0550-3213(84)90066-X

[40] S. Deguchi and K. Fujikawa, Phys. Rev. A 72, 012111 (2005) [hep-th/0501166].

[41] K. Fujikawa, arXiv:1709.08181 [hep-th].

[42] N. Mueller and R. Venugopalan, in preparation. 